\title{
Co-factors and HIV: What Determines the Pathogenesis of AIDS?
}

\author{
June E. Osborn
}

\begin{abstract}
Microbiology and infectious disease are replete with examples of 'the iceberg phenomenon' which, simply put, describes the fact that for every person suffering disease from a given microorganism, a number of others are infected but do not suffer the characteristic illness ascribed to that pathogen. This variable expression of pathogenic properties is true of all but a few microorganisms of man, and the newly identified virus HIV, associated with AIDS, will in fact be exceptional if it uniformly initiates the destructive processes which set the stage for immunosuppression and AIDS.
\end{abstract}

It is well appreciated that a number of ' risk factors' help predict with a high degree of accuracy which persons are likely to become infected with HIV, ${ }^{1}$ a virus which is fortunately so fastidious and fragile that its spread among humans depends almost exclusively on sexual, blood or perinatal transmission. Within groups of infected individuals (that is, persons who have antibody to HIV), it is still uncertain who will develop AIDS or related diseases, but prospective studies are being pursued vigorously in hopes of identifying specific environmental or behavioral components of life style that might prove predictive of good or bad outcomes of established HIV infections. The range of possibilities is wide: there have been individuals infected since earliest recognition (1978) of the U.S. epidemic who remain healthy, albeit infected and infectious eight years later. Conversely, some AIDS patients have sickened and died in less than a year; and in the highest known seropositive risk groups, the rate of AIDS occurring after three years' infection is less than $50 \% .^{2}$ Gloom pervades the estimates of the future of these seropositive 'worried well' but they may not hold the full key to the intrinsic pathogenicity of AIDS, for they are far from the norm in many ways which may have bearing: that is to say, a number of potential 'co-factors' are intrinsic to their membership in risk groups to start with.

Perhaps the simplest example would be that of the severe hemophiliacs who were dependent on factor VIII concen- trate obtained from plasmapheresis donors for their hemostasis and thus were squarely in the path of the new virus when it entered the U.S. blood supply prior to 1983. Factor VIII concentrate is prepared from the pooled plasma of literally thousands of donors, and the virus was evidently able to survive the prevailing fractionation procedures, so that over $90 \%$ of the cohort of severe hemophiliacs became seropositive prior to the institution of effective control measures (donor selfexclusion, heat-treatment of concentrate and donor antibody screening). These hemophiliacs were important 'sentinels' of the blood supply, and intensive studies were launched prior to recognition of the new virus, looking for explanations of their involvement in the burgeoning epidemic. In this context it was recognized that they were being bombarded with foreign antigens by virtue of their factor VIII treatment and were, in fact, measurably immunosuppressed prior to virus infection. ${ }^{3}$ This helped to give rise to the theory of antigenic overload as a possible mechanism of AIDS, and while the discovery of the new virus ablated that theory as a self-contained explanation of AIDS, antigenic overload remains as a possible co-factor which might augment or accelerate the pathogenesis of immune deficiency.

Hemophiliacs present the mildest and simplect examples of this kind of postulated co-factor: other than the alloantigens represented in factor VIII their microbial and social environment is relatively commonplace, and it is of interest to note that they are at once the highest risk group for HIV infection and the lowest (among the risk groups) for AIDS. ${ }^{2}$

In terms of antigenic stimulation and overload, the other risk groups present much more dramatic examples. Gay men with multiple sexual partners have multiple infectious diseases, for parasites, bacteria and a variety of viruses thrive in the context of sexually active gay life styles. Similarly, i.v. drug users bombard themselves not only with HIV (in the act of needle-sharing) but also are notorious for their plethora of infections such as the viral hepatitides and bacterial endocarditis. Haitians, whose role early in the epidemic was inexplicable but significant, share their terrain with a wide variety of parasites, suffer from endemic tubercular infections, and again conform to the pattern of potential antigenic overstimulation.

The mechanism whereby these cofactors might play an additive or synergistic role with HIV is not hard to postulate: the proliferation of T4 helper cells at the initiation of the immune response would at the very least put a 'stretch' on a weakened immunologic capability; but it also might activate HIV simultaneously with amplification of the susceptible T4 helper cell substrate, for which the virus clearly has both a taste and a lytic potential. ${ }^{4}$ Thus a vicious circle of viral replication and enlargement of the pool of susceptible cells is easily imagined, perhaps reaching a point of no return with irretrievable lysis-mediated depletion of T4 helper cells - the immunologic setting for the opportunistic infections diagnostic of AIDS.

Another co-factorial mechanism which might be operative in the progression from asymptomatic infection to overt disease is coincident immune suppression caused by factors other than HIV: a number of viruses prevalent among gay men have transient immunosuppressive effects of their own-notably cytomegalovirus ${ }^{5}$ and Epstein-Barr virus ${ }^{6}$ - and an unhappy coincidence of timing might result in irreversible damage to the immune system. Similarly, semen has immunosuppressive effects, ${ }^{7}$ as do a number of drugs of abuse (marijuana as well as the opiates and nitrites).

Another kind of immunosuppression corresponds in an interesting way to an already-established co-factor of substantial risk: i.e. pregnancy. It is worth reviewing briefly the information concerning the adverse effect of pregnancy on asymptomatic HIV infection, for while the numbers are small they are dramatic. In a study in Miami, Scott and colleagues initially identified several infected women by the occurrence of AIDS in their infants. ${ }^{8}$ Of these asymp- 


\section{ChALLENGeS}

tomatic women, 13 subsequently became pregnant and delivered 15 second children who could be followed. By one year of age only 4 infants were uninfected, one was asymptomatically infected, five had immunologic abnormalities and 5 had AIDS with an average age of onset of 4 months. The extent of the adverse effect on these offspring was unexpected, in that it is nearly unique among congenital viral infections to feature successive, pathologically significant intra-uterine infections. However, the most striking finding of the study was that more than half the mothers sickened and four had died of AIDS within a very few months of the second delivery - a pace and rate of exacerbation greater than had been recognized in any other high-risk cohort.

This effect of pregnancy on the virus-host relationship is worthy of study. It is a fascinating aspect of immunology that gestational immunosuppression occurs to some extent; in the second and third timesters one can measure depressed lymphocyte numbers and functions which generally return to normal by one month post-partum. ${ }^{9}$ That this should occur is intuitively reasonable since the maternal environment must remain tolerant to alien antigens for many months. There may be clues to the specific synergism between pregnancy and HIV which are useful in providing broader insight about the pathogenesis of AIDS; but for the present it constitutes a strongly documented warning that pregnancy may be very dangerous to the health of an infected woman.

As a final possible example of additive immune suppression it is evident that superinfection with more than one variant of HIV can occur. ${ }^{10}$ The potential of this mechanism for saturating (and destroying) susceptible cells has not been assessed, but the continued intercourse among multiple HIV-positive sexual partners may carry incremental risks. Note that to the extent that this superficial infection phenomenon does occur, the hoped-for vaccine strategy of utilizing interfering retroviruses such as HIV-II ${ }^{11}$ may be less promising than would at first appear.

Up to this point, the discussion has focused on individuals who were inherently 'normal' at least at the time of acquisition of HIV. It is noteworthy, however, that several risk groups are composed of people who were not immunologically normal at time of first exposure. It has been suggested, for instance, that the different distribution of AIDS between the sexes in central
Africa (relative to this distribution in other countries) may reflect a different human substrate for the virus. ${ }^{12}$ The most common factor cited in this argument is the underlying immunosuppression attendant on malarial prevalence in those regions in which AIDS is now epidemic. This line of reasoning is almost exactly parallel to that proposed to explain the relationship between Burkitt's lymphoma and Epstein-Barr virus, and it is certainly an interesting avenue to explore, since the equal male-female distribution in African patients is a source of nervous worry lest it represent a premonitory change in the nature of HIV itself. There are actually a number of alternative explanations for the divergent African experience with HIV, however, including patterns of needle use in clinics where sexually transmitted diseases are treated, ${ }^{13}$ and practices of clitorectomy which might either provide relatively easy access to the bloodstream during vaginal intercourse or alter patterns of heterosexual practice. Unfortunately these matters are caught up in a rather heated cross-cultural debate, and it may take a while for tempers to cool and sensitivities to ease enough to permit progress in understanding the divergent epidemiology of AIDS in Africa. ${ }^{14}$

Infants are another immunologically abnormal group, and the more premature they are, the greater their immunological abnormality. The significant shortening of average incubation interval in AIDS infants from 2 or more years to less than a year has been explained on that basis, at least in a general way. Furthermore, those infants who acquired their viral infection through multiple blood transfusions could also be viewed as candidates for antigenic overload, in view of the abundance of foreign histocompatibility antigens represented in the repeated transfusions.

Another kind of immunologic abnormality worthy of considerable concern is that ascribed to the process of aging. It has been shown that there is - or at least can be - a significant attrition of immunologic response capabilities in the seventh and later decades. This is probably not trivial, as illustrated by the well-known fact that the risk of herpes zoster (or so-called shingles) goes up precipitously with advancing age. While this age group is not represented at present in the population of asymptomatic, infected persons, it may loom large as a co-factor when that cohort ages.

To this point the discussion has centred on HIV and/or direct observations from experience with the AIDS epidemic which suggest avenues of explanation for variable clinical outcome. However, it is interesting and perhaps useful to look at other diseases in which clinical expression seems to be the summation of disparate stimuli. Perhaps the most pertinent would be the area of latency and reactivation of herpes simplex. The link between emotional states and exacerbation of cold sores, for instance, is well accepted, and yet intense study directed to the establishment and maintenance of herpes simplex latency has as yet yielded no compelling insight to explain such connections to emotional and neurobehavioral events. Insights in this area could well have substantial bearing on the pathogenesis of AIDS.

In a similar vein, there is an even less satisfactory but persistent association between acute emotional perturbation and the abrupt onset of malignancy, which might be a related phenomenon, if it is true that the immune system is constantly exercising a surveillance function to minitor for abnormal cells. If the immunologic component of grief reactions, for instance, is a contributor to the progression of symptomatic disease in the context of AIDS, it will be critically important to learn that and to deal with it in counselling of infected persons.

In summary, then, there is no shortage of co-factors to be considered as possible contributors to disease onset and progression in the AIDS epidemic The urgency of their assessment is manifest in the fact that an estimated 1-2 million asymptomatic individuals in the United States alone now live under the potential threat of a lethal disease, ${ }^{15}$ and the likelihood of that occurring may be not only influenced but actually determined by specific co-factors. Some of them-such as pregnancy, immunosuppressive drugs, and exposure to multiple infectious agents - may be fairly easy to control if we understand their significance well enought to justify the effort. Many of them submit to health education strategies in a manner quite analogous to counselling concerning juvenile-onset diabetes. In fact, the diabetes analogy is quite useful in thinking about this problem. In that instance the new juvenile diabetic must learn to live with the realization that his islet cells are not to be relied upon and that life-style changes will be necessary; but once those accommodations are made, he can anticipate relatively good health 
CHALLENGES

and longevity. In the instance of HIV infection, there is room for hope that the same concept applies to T4 helper cells: they, too, are weakened and fragile, but with enough insight about determinants of progression in the context of virus infection, it may be possible through health education to define life styles which facilitate continued well-being for many years despite the lurking threat of viral reactivation. As the shadow of HIV infection grows longer and as the likelihood of treatment strategies remains ephemeral, these avenues of investigation may promise solid hope for the health of a very large number of infected persons and are worthy of intensive pursuit.

\section{REFERENCES}

1 Coffin, J., HaAse, A., Levy, J. A., Montagnier, L., Oroszlan, S., Teich, N., Temin, H., Toyoshima, K., Varmus, H., VoGT, P. \& WeISS, R. (1986). Human immunodeficiency viruses (letter). Science 232, 697.

2 Goedert, J. J., Bigger, R. J., Weiss, S. H., Eyster, M. E., Melbye, M., Wilson, S., GinzburG, H. M., Grossman, R. J., DiGiola, R. A., Sanchez, W. C., Giron, J. A., Ebbesen, P., Gallo, R. C. \& BlattNER, W. A. (1986). Three-year incidence of AIDS in five cohorts of HTLV-IIIinfected risk group members. Science 231 , 992-995.
3 Shannon, B. T., Roach, J., CheekLuten, M. \& RUYManN, F. B. (1986). HTLV-III status and abnormalities in T lymphocyte distribution in children with hemophilia A. Diagnostic Immunol. 4, 37-42.

4 Bowen, D. L., Lane, H. C. \& Fauci, A. S. (1985). Immunologic features of AIDS. In AIDS: Etiology, Diagnosis, Treatment and Prevention (ed. V. T. DeVita, Jr, S. Hellman \& S. A. Rosenberg) Lippincott, New York.

5 Carney, W. P. \& Hirsch, M. S. (1981). Mechanisms of immunosuppression in cytomegalovirus mononucleosis. J. Inf. Dis. 144, 47-54.

6 Bowen, T. J., WEDGeWOOD, R. J., OCHS, H. D. \& Henle, W. (1983). Transient immunodeficiency during asymptomatic Epstein-Barr virus infection. Pediatrics 71, 964-966.

7 Kuno, S., Ueno, R., Hayaishi, P., Nakashima, J., Harada, S. \& Yamamoto, N. (1986). Prostaglandin $E_{2}$, a seminal constituent, facilitates the replication of acquired immune deficiency syndrome virus in vitro. Proc. Nat. Acad. Sci. USA 83, 3487-3490.

8 Scott, G. B., Fischl, M. A., Klimas, N., Feltcher, M. A., Dickenson, G. M., Levine, R. S. \& Parks, W. P. (1985). Mothers of infants with the acquired immunodeficiency syndrome. JAMA 252, 363-366.

9 Tallon, D. F., Darach Corcoran, D. J., O'Dwyer, E. M. \& Greally, J. F. (1984). Circulating lymphocyte subpopulations in pregnancy: a longitudinal study. J. Immunology 132, 1784-1787.
10 Wong-Staal, F., Shaw, G. M., Hahn, B. H., Salahuddin, S. Z., Popovic, M., Markham, P., Redfield, R. \& Gallo, R. C. (1985). Genomic diversity of human T-lymphotropic virus types III (HTLV-III). Science 229, 759-762.

11 Kanki, P. J., Barin, F., M'Boup, S., Allan, J. S., Romet-Lemonne, J. L., MarLINK, R., MCLANE, M. F., LEe, T.-H., Arbeille, B., Denis, F. \& Essex, M. (1986). New human T-lymphotropic retrovirus related to simian $\mathrm{T}$-lymphotropic virus type III (STLV-III AGM $_{\text {). Science 232, 238-243. }}$.

12 Biggar, R. J. (1986). The AIDS problem in Africa. Lancet i, 79-83.

13 Kreiss, J. K. Koech, D., Plummer, F. A., Holmes, K. K., LightFOOTE, M., Piot, P., Ronald, A. R., Ndinya-Achola, J. O., D'Costa, L. J., Roberts, P., NGugi, E. N. \& QuinN, T. C. (1986). AIDS virus infection in Nairobi prostitutes: spread of the epidemic to East Africa. New Eng. $J$. Med. 314, 414-418.

14 Norman, C. (1986). Politics and science clash on African AIDS. Science 230, 1140-1141.

15 Barnes, D. M. (1986). Grim projections for AIDS epidemic. Science 232, 1589-90.

JUNE E. OSBORN is Dean of the School of Public Health, The University of Michigan, 109 South Observatory St, Ann Arbor, Michigan 48109, USA.

\section{CORRESPONDENCE}

BioEssays welcomes comments from readers on articles published in the journal or on related matters. Correspondence in the form of letters to the editor should be addressed either to $\mathrm{Dr}$ W. J. Whelan, Editor-in-Chief, BioEssays, Biochemistry, P.O. Box 016129, Miami, Florida 33101, U.S.A., or to Dr A. S. Wilkins, Staff Editor, BioEssays, Cambridge University Press, Edinburgh Building, Shaftesbury Rd., Cambridge CB2 2RU, England.

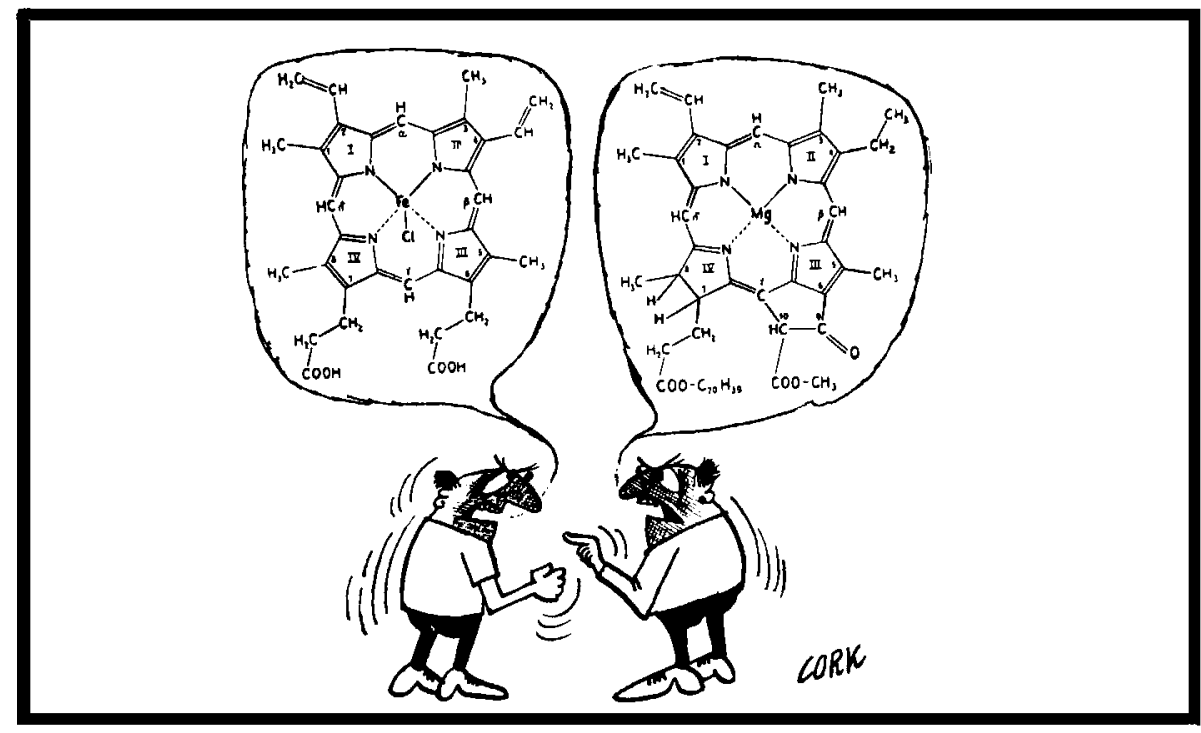

